\title{
Reduction of dimensional variability of pieces, resulted in AWJ process, using robust design method
}

\author{
Bogdan Barabas ${ }^{1, *}$, Tudor Deaconescu ${ }^{1}$, and Sorin Barabas ${ }^{1}$ \\ ${ }^{1}$ Transilvania University of Brasov, Faculty of Technological Engineering and Industrial \\ Management, Department of Engineering and Industrial Management, Brasov, Romania
}

\begin{abstract}
Dimensional variability arises in AWJ system from uncontrolled factors intervention or due to the variability of input signals considered fixed or due to interactions of the input signals with unexpected effects, leading to deterioration of unpredictable outcome. Number of noise factors, negatively influence the results registered, dispersion of the result values is directly proportional to the number and their effect. Stochastic influence and his unpredictable nature determine a thorough research of the hydroabrasive process. The research refers to the study of dimensional variability for different configurations of hydroabrasive liquid pressure, abrasive type and traverse velocity. This is achieved by performing the experiments statistically processed by combining of control parameters, which allow favorable conclusions on reducing variability results and on optimizing the entire process of hydroabrasive machining.
\end{abstract}

\section{Introduction}

Abrasive water jet (AWJ) erosion is an extremely complex system wherein the plurality of parameters that determine the phenomena occurring at extremely high pressures, stochastic responses resulted, are leading to the need of using probability and statistical calculation for the study of this processing methods. Robust design method of systems that rely on the use of instruments mentioned above may contribute decisively to the mathematical modelling of processing technology by hydro-abrasive jet.

Using robust design in optimization of hydro-abrasive jet processing consists in choosing of an optimal combination of parameters that influence the process, so that it becomes insensitive to sources of variability, and the result obtained is maximal in terms of quality [1]. Dispersion values reduction is aimed and their focusing on a target value, tending to constant values by judiciously combining controlled parameters that define the system.

Advantages of a robust approach of hydro-abrasive jet processing optimization consist in the possibility of studying the dynamic of the whole factors ensemble, being in interdependence. Applying robust design on the technological process of hydro-abrasive jet

\footnotetext{
${ }^{*}$ Corresponding author: barabas.bogdan.florin@unitbv.ro
} 
processing is achieved through determining noise factors $z$ and preventing the propagation of their stochastic variation effects on a system $y[2]$.

$$
y=f(x, z)
$$

where $x$ represents values of controlled parameters of technological system. It is noted that the values of system responses, y, are also random. Developing the relationship (1) as a Taylor series, lead to expression:

$$
f(x, z)=f(x, \mu z)+f^{\prime}(x, \mu z)(z-\mu z)+\frac{f^{\prime \prime}(x, \mu z)}{2 !}(z-\mu z)^{2}
$$

where $\mu$ is average of stochastic variables $z$. Equation (2) shows the influence of variable $z$ on system $y$, respectively on transfer function $f$ that represent AWJ system. Average of variance of answers given by system y is calculated using the equation (3):

$$
\operatorname{Var}(y)=\frac{1}{n} \sum_{i=1}^{n}\left(x_{i}-R_{y}\right)^{2}
$$

where $n$ represents the number of random values system responses $\mathrm{y} ; x_{i}$ represents values of controlled factors of system and $R_{y}$ represents the average of those values.

$$
R(y)=R[f(x, z)] \approx f\left(x, \mu_{z}\right)+f^{\prime}\left(x, \mu_{z}\right) R\left(z-\mu_{z}\right)=f\left(x, \mu_{z}\right)
$$

Of (3) and (4) equations, resulting Gauss approximation formula:

$$
\operatorname{Var}(y)=\operatorname{Var}[f(x, z)] \approx \operatorname{Var}\left[f^{\prime}\left(x, \mu_{z}\right)\left(z-\mu_{z}\right)\right]=\left[f^{\prime}\left(x, \mu_{z}\right)\right]^{2} \sigma_{z}^{2}
$$

where $\mu_{z}$ represent standard deviation for values of answers of system y for values variation of stochastics factors $z$.

For a hydro-abrasive jet processing system, $z$ is the noise factor (variable which can't be controlled completely), and $x$ is the factor on which there is total control. The system responses $y$ can be, where appropriate, cut surface roughness, processing cost, processing time, or other response. They may be controlled, their dispersion can be minimized, leading to a target value, by using the Gauss's approximation [3]:

$$
\begin{aligned}
& \mu_{y} \approx f\left(\mu_{z}\right) \\
& \sigma_{y}^{2} \approx\left[f^{\prime}\left(\mu_{z}\right)\right]^{2} \sigma_{z}^{2}
\end{aligned}
$$

where $\mu_{z}$ și $\sigma_{y}$ represent average, respectively standard deviation for values of measured answers.

Optimization of technological process of hydro-abrasive jet processing is achieved by determining the parameters, their type and the variation interval of values [4].

\section{Optimizing hydroabrasive jet processing by reducing the variability of responses}

The variation of responses by hydro-abrasive erosion processing system is characterized by a measurable error that occurs from one piece to another, even if it retains control the same controlled parameters. This variation may be due to: unforeseen interactions of control factors; unexpected changes in any controlled parameter; environment (temperature, humidity fluctuations); user. 
The difference between the obtained response and the expected response is the response error, which is a nonlinear function related to entries into the system, controlled parameters and uncontrollable factors (noises) and interactions occurring [5-6]

Errors that occur from one piece to another, although they are executed under the same conditions can be mitigated and managed to a minimum, thus optimizing the entire technological process.

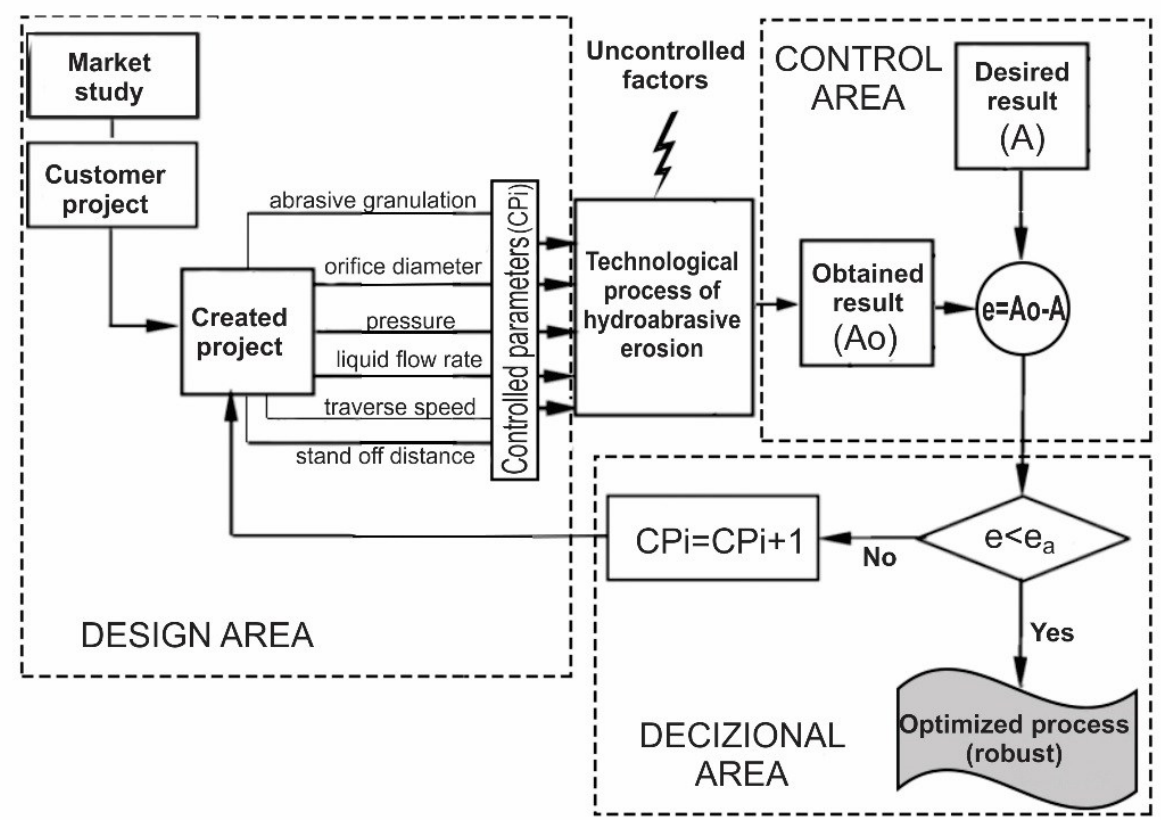

Fig. 1. Algorithm for optimization of erosion hydroabrasive process

Arrangement of controlled parameters so that whatever action of uncontrollable factors is, the difference between the desired result and the obtained result $\left(e=A_{o}-A\right)$ is less than a permissible variation $e_{a}$ conducting to robust results. If $e>e_{a}$, then move to a new combination of controlled factors until the algorithm will generate a controlled parameters solution that insensitize the system to uncontrolled actions and influences.

Considering, for example, a tolerated dimension the objective of optimizing the hydroabrasive process, algorithm from Fig. 1, allow the following assertion:

$$
\text { Optim }=\operatorname{Minim}\left|A_{0}\left(C P_{i}\right)-A\left(C P_{i}\right)\right|, A_{0}\left(C P_{i}\right) \in[a, b]
$$

where: $A_{0}$ is obtained dimension $[\mathrm{mm}]$; $A$ is desired dimension $[\mathrm{mm}]$; $\mathrm{CP}_{\mathrm{i}}$ represents controlled parameters setup; $[\mathrm{a}, \mathrm{b}]$ represents tolerated dimensional interval accepted for nominal dimension A.

Optimisation is realized by minimizing the error and by insensitivity of results to any other influence outside that resulting to controlled parameters. Average error $\mathrm{e}_{\mathrm{med}}$, caused by noise, becomes:

$$
e_{\text {med }}=\frac{A_{o 1}+A_{o 2}+\cdots+A_{o i}}{i}-A=\mu_{A o i}-A
$$

By applying the relationships (8) and (9):

$$
\text { Optim }=\operatorname{Minim}\left|\mu_{A o i}-A\right|, A_{0}\left(C P_{i}\right) \in[a, b]
$$




\section{Determination of experiments plans}

Reducing the dimensional variation of the pieces resulting from processing with hydroabrasive jet is quantified by minimizing the difference between the obtained dimension $A_{0}$ and nominal dimension, $A$ (10) [7]. The formula used for minimization of dimensional variation is calculated using minimum effects caused by chosen parameters

$$
e=e_{m e d}+\sum_{i=1}^{n} \min E_{i}
$$

where: $-e$ is difference between effective obtained size and nominal size[mm]; $e_{m e d}$ is arithmetic average of the measured $e$ in all experiments $[\mathrm{mm}] ; E_{i}$ is the effect of each combination of parameters.

For verification was used Taguchi method [8, 9]. The optimal combination of parameters is made using criterion of minimization of signal-noise ratio:

$$
\frac{S}{N}=-10 \log \left(s^{2}+\bar{y}^{2}\right)
$$

where: - $S / N$ is ratios depending of parameters values (performance indicator); $s$ is standard deviation of data; $y$ is arithmetic average of data.

\subsection{Configuration of parameters that influence the hydroabrasive processing}

Setting of processing with hydroabrasive jet was applied on a machine Maxiem Jetcutting center 1530. Were used high pressure with following values: $p_{1}=345 \mathrm{MPa}$ and $p_{2}=180$ MPa. Were used two types of garnet abrasive: Mesh 80 and Mesh 100, with following characteristics (Table 1).

Table 1. Properties of used abrasives

\begin{tabular}{|r|c|c|c|c|c|}
\hline No. & Type of abrasive & Grain size $[\mathbf{m m}]$ & Density $\left[\mathbf{g} / \mathbf{c m}^{3}\right]$ & Hardness [Mohs] & Purity \\
\hline 1. & Garnet Mesh 80 & 0.18 & 2.31 & $>8$ & $>80 \%$ \\
\hline 2. & Garnet Mesh 100 & 0.15 & 2.40 & $>8$ & $>80 \%$ \\
\hline
\end{tabular}

The used material is alloyed austenitic stainless steel $\mathrm{X} 5 \mathrm{CrNi} 18-10$ with following properties: Hardness Vickers, $\mathrm{HV}=190$; machinability, $\mathrm{M}=80.8$; modulus of elasticity, $\mathrm{E}$ $=200 \mathrm{GPa}$. Samples consisted in execution of rectangles with thickness $\mathrm{H}=10 \mathrm{~mm}$, the measured nominal dimension, $A=20 \mathrm{~mm}$ and $\mathrm{B}=50 \mathrm{~mm}$. Five samples were achieved for each combination of factors, overall executed 40 samples.

The used software for AWJ setting and cutting was Intelli-Max Make, version 23.0. In Table 2 is shown the two levels of variance for input parameters.

Table 2. Parameters values used in experiments

\begin{tabular}{|c|c|c|c|c|c|}
\hline Parameters & $\begin{array}{c}\text { Traverse } \\
\text { speed } \\
{[\mathbf{m m} / \mathbf{m i n}]}\end{array}$ & $\begin{array}{c}\text { Pressure } \\
{[\mathbf{M P a}]}\end{array}$ & $\begin{array}{c}\text { Stand-off } \\
\text { distance } \\
{[\mathbf{m m}]}\end{array}$ & $\begin{array}{c}\text { Type of } \\
\text { garnet } \\
{[\mathbf{M e s h}]}\end{array}$ & $\begin{array}{c}\text { Abrasive } \\
\text { mass flow } \\
{[\mathbf{K g} / \mathbf{m i n}]}\end{array}$ \\
\hline & $\mathbf{A}$ & $\mathbf{B}$ & $\mathbf{C}$ & $\mathbf{D}$ & $\mathbf{E}$ \\
\hline Level 1 & 60 & 180 & 1 & 80 & 0.05 \\
\hline Level 2 & 90 & 345 & 2 & 100 & 0.08 \\
\hline
\end{tabular}




\subsection{Experimental results}

The plan of experiments is presented in Table 3. Obtained dimension $\mathrm{A}_{0}$ is measured and in table is written the difference to the desired dimension A, considered theoretical dimension, by calibrating the software. Combinations of configurations parameters are influenced differently by uncontrollable noise factors [10-11].

Table 3. Matrix of experiments and their results

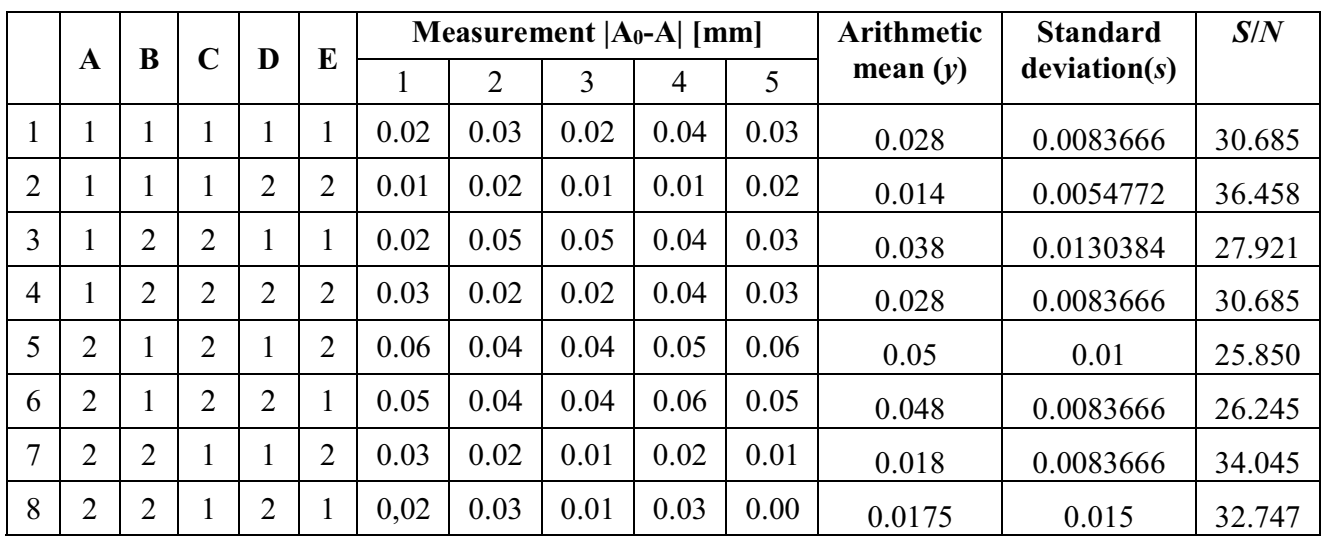

In classical method is calculated the average response for each factor and for each level. The calculated responses is shown in Table 4, where in row 1 is average response and in row 2 is shown the average effect.

Table 4. The average response and calculated effect of combined parameters

\begin{tabular}{|c|c|c|c|c|c|c|c|c|c|c|}
\hline & A1 & A2 & B1 & B2 & C1 & C2 & D1 & D2 & E1 & E2 \\
\hline 1 & 0.027 & 0.03337 & 0.035 & 0.02537 & 0.0193 & 0.041 & 0.0335 & 0.0269 & 0.03287 & 0.0275 \\
\hline 2 & -0.003 & 0.003 & 0.005 & -0.005 & -0.011 & 0.011 & 0.003 & -0.003 & 0.0027 & -0.0027 \\
\hline
\end{tabular}

Using equation (11) is obtained $\mathrm{e}_{\mathrm{min}}=\left|\mathrm{A}_{0}-\mathrm{A}\right|=0.006[\mathrm{~mm}]$ and parameters value combination: 12122 (Fig.2).

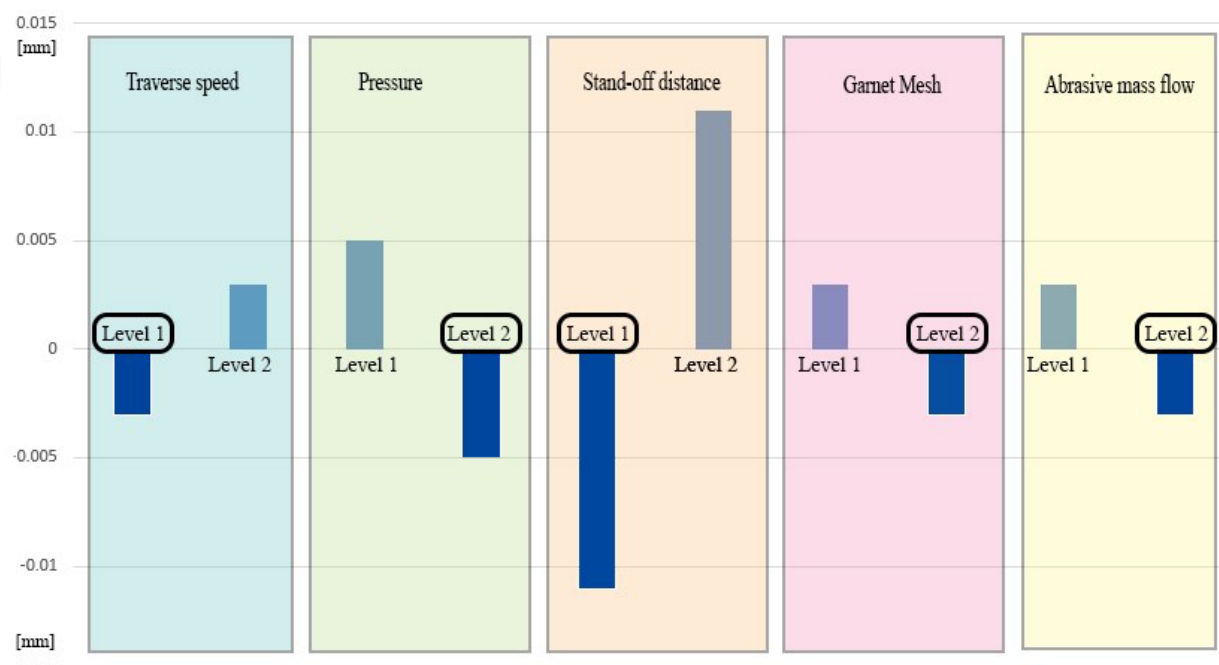

Fig. 2. Effect of different levels of parameters on dimensional variability in AWJ process 
With Taguchi method is calculated the response for every ratio $S / N$ and the resulted effect shown in Table 5, where in row 1 is average $\mathrm{S} / \mathrm{N}$ response and in row 2 is shown the average effect.

Table 5. The average response of $\mathrm{S} / \mathrm{N}$ and calculated effect of combined parameters

\begin{tabular}{|c|c|c|c|c|c|c|c|c|c|c|}
\hline & A1 & A2 & B1 & B2 & C1 & C2 & D1 & D2 & E1 & E2 \\
\hline 1 & 31.437 & 29.721 & 29.809 & 31.349 & 33.484 & 27.675 & 29.625 & 31.534 & 29.399 & 31.759 \\
\hline 2 & 0.857 & -0.857 & -0.769 & 0.769 & 2.904 & -2.904 & -0.954 & 0.954 & -1.180 & 1.180 \\
\hline
\end{tabular}

With Taguchi method, the optimal combination of input parameters is 12122 .

\section{Conclusions}

Were executed 40 samples which consisted in cutting rectangles from steel plate $\mathrm{X} 5 \mathrm{CrNi}$ 18-10, with thickness $\mathrm{H}=10 \mathrm{~mm}$. Were chosen 5 parameters with notable influence on dimensional precision, receiving 2 values, each parameter. To the 5 parameters with 2 levels, was attributed matrix of plans of experiments from tab. 3 and for each combination of parameters were performed 5 measurements, introducing in tab.3, the arithmetic mean and standard deviation. The signal-noise report was calculated with relation (12) for minimization of dimensional variability.

The arithmetic average of all measurements taken, representing the difference in absolute value, compared to the nominal size was $\mathrm{e}_{\mathrm{med}}=0.03 \mathrm{~mm}$, equivalent to a tolerance field, equal with $0.06 \mathrm{~mm}$. For certain combinations, the tolerance field was situated at 0.1 $\mathrm{mm}$ (21212). In Fig. 2 it is observed that certain levels of parameters, influences dimensional variability in the sense of magnifications of tolerance field, and other levels reduces tolerance field. Choosing those levels which minimizes the tolerance field, implicitly dimensional variability is obtained according to (11) $\mathrm{e}_{\min }=0.006 \mathrm{~mm}$, equivalent with a tolerance field equal with $0.012 \mathrm{~mm}$. Using plans of experiments, allows choosing a combination of parameters, that, even if was not used in performing tests, it is the optimal combination resulting in reduced variability and increased dimensional accuracy of execution.

\section{References}

1. V. Sharma, S. Chattopadhyaya, S. Hloch, Int J Adv Manuf Technol 56, 1019-1025 (2011)

2. M.Arner, Statistical robust design, (Wiley, 2014)

3. J. Kechagias, G. Petropoulos, N. Vaxevanidis, Int J Adv Manuf Technol 62, 635-643 (2012)

4. C. W. Kang, P. H. Kvam, Basic Statistical Tools for Improving Quality, (Wiley 19-25, 2011)

5. D. K. Shanmugam, S. H. Masood, J. Mater. Process Technol. 209, 3887-3893 (2009)

6. A. Perec, Proc.Eng., 149, 366-373 (2016)

7. D. Trainer, Int., J. Multi. Flow, 81, 77-87 (2016)

8. A. Radvanska, T. Ergic, Z. Ivandic, S. Hloch, J. Valicek, J. Mullerova, Strojarstvo 51, 347-354 (2009)

9. G. Taguchi, S. Chowdhury, Y. Wu, Taguchi's Quality Engineering Handbook, (Wiley, 239-263, 2005)

10. R. Shukla, D. Singh, Swarm and Ev. Comp. (to be published)

11. Jagadish, S. Bhowmik, A. Ray, Int J Adv Manuf Technol 87, 1359-1370 (2016) 